\title{
New Approach in Investigating the Role of Interaction between an Organism and Physical Factors in Complex Patient Therapy
}

\author{
Vladmimir Dodtievich Bitsoev \\ Doctor of Medical Science, h.42A, Kirova str., apt. 60 the town of Podolsk of the Moscow Region, Moscow, Russia \\ Email: bitsoev@mail.ru
}

Received 10 March 2016; accepted 24 March 2016; published 29 March 2016

Copyright (C) 2016 by author and OALib.

This work is licensed under the Creative Commons Attribution International License (CC BY). http://creativecommons.org/licenses/by/4.0/

(c) (†) Open Access

\begin{abstract}
This work is dedicated to the new approach in applying physical factors in complex patient therapy. Based on experimental research data the author believes it is necessary to reconsider technical characteristics and exposition of physiotherapeutic factor influencing on the organism taking into account nosological form of disease and individual patient's specifics. In this regard the author developed new tasks of the clinical physiotherapy and suggested seven principles of new classification of physical factors in complex patient therapy. On the basis of the research the author provided new concepts in medicine: 1) human organism as "biological scanning tunneling microscope"; 2) human as a part of "Nature" corresponds to universe general law: "singularity and indivisibility of time, matter and form", as well as the forces of their interaction under constant endless motion.
\end{abstract}

\section{Keywords}

Evanescent Waves, Solitons, Biological Scanning Tunneling Photonic Microscope

Subject Areas: Clinical Trials

\section{Introduction}

Rational use of physical factors in complex patient therapy follows the principle of strictly differentiated selection of used energy type and their order of implementation which fully corresponds to clinical presentation.

Clinical presentation of any nosological disease form for a particular patient is presented with an absolutely individual range of symptoms as a result of complex ethiopathogenetic process, depending on the varieties of negative internal and external factors.

Although today doctors have thousands of pharmacological drugs available, most of them are either insuffi- 
ciently effective or possess side effects. Physical factors (of low intensity) not only do not cause any allergic reactions but also have antiallergic immune-corrective effect [1].

Cells are capable of converting a strictly limited amount or a certain range of any physical factor's energy into its specific biological process. Energy is increasingly beyond the scope of absorbing range (individual for all types of cells), cells are blocked and bypass (more favorable option) or undergo destruction during the breakdown of protective cellular block. Maximum energy in extremely small space represents the danger of destruction whereas maximum space with minimum energy at each point represents the characteristic of life evolution (A. Zalmanov, 1991).

This concept makes it possible to do the following:

1. Studying the leading characteristics-frequency, wave length, power, cyclicity of cell bioenergy under normal and pathological conditions.

2. Determining the degree of disorders in specific biological processes of organs and systems.

3. Choosing medical physical factors (energy type, parameters, individual method, course of treatment) to recover disordered biological processes.

In our opinion, the fact that the diseased cells are not able to obtain and convert such intensive energy into their specific biological process is possibly one of the sources of pathological process of complications and side effects. This can also unbalance other organs and systems.

Since the really acting and absorbing energy on the living cell is presented with minimum physical factor characteristics with respect to integral organism whereas at cellular level it is presented as intensive therapeutic influence [1].

There are no two identical elementary particles existing in the Nature. Therefore, the components that are made up of them—-from subcellular supramolecular formations to the space itself-do not exist either. This is the main condition for everlasting endless constant motion and Universal eternity.

Taking the example of a living organism in any system (muscular, circulatory, nervous, cardiovascular, etc.) there are no two cells with identical matter or form since, otherwise, there would be no muscle contraction, momentum transfer, cellicolous and intercellular motion, i.e. there would be a complete absence of living.

A human being is a complex nonlinear system composed of a large number of different functional structures. Nervous, endocrine and pulse systems maintain informational balance within the organism, modeling and compensating mutual signal influences. There is a developed concept of "communicatory-and-regulatory apparatus which operates in two ways: conductive, with electrical signal transmission (nerve cells) and humoral, based on the transport of various bioregulators (nerve, mesenchymal and epithelial cells)" [1]-[3].

Cellicolous structure of any system-and, therefore, cellicolous energy-is unique. For that reason there is variation of intercellular energy - the source of constant endless motion with a vast infinite range of speeds which creates process cyclicity of "matter-energy" transitions from one state to another. That is the eternal existence of the Universe. However our capabilities of its understanding are restricted within the scope of human knowledge.

In her "Knocking on Heaven's Door" book a professor of Harward university, a remarkable American theoretical physicist and a competent specialist in the field of elementary-particle physics Lisa Rendall notes that "studying the increasingly short inter-particle distances is interesting due to the fact that in this world rules may change with changes in scope. Totally new interactions and forces may be displayed. Their effects cannot be captured at the previous level of research i.e. at larger distances.

The idea of scope which tells the physicist about the dimensions and energies concerned in this research is very important for understanding scientific progress and numerous other aspects of the surrounding world. The research showed that the same principles of physics by no means play the key role in every process" [4].

This is the confirmation of our concept: "every cell of the surrounding world is unique in its matter and form. For this reason there is an eternally cellicolous, intercellular and intersystem energy which creates the infinitely endless motion i.e. the speed of eternal transition of matter-energy from one state to another which is established by the law of nature.

Lisa Rendall further writes in her book: "in physics they categorize objects based on their size in order to have the possibility to focus on the points of our interest. For example, it seems to us that the top of a writing table is hard - when it is hard, indeed - but it is virtually composed of atoms and molecules. It is them that altogether act as a hard non-intrusive surface which we deal with every day. But atoms and molecules themselves do not represent indivisible matter either; each atom contains a nucleus and electrons. And an atomic nucleus is composed of protons and neutrons which, in turn, represent more fundamental objects known as quarks. 
And it is still not necessary to be aware of the quarks in order to understand electrical-and-magnetic and chemical features of atoms and chemical elements (this is studied by nuclear physics)" [4].

In these thoughts of professor Lisa Rendall we also find the confirmation of our concept that the surrounding world is composed of infinitely unique combinations of quarks as a source of creating extremely unique energy of these combinations which make up the whole World, from cells to the Space. Therefore, there is always variation of energy among any cells, formations, etc.-a source of endless motion.

Due to the existing endless motion of the Entire surrounding World there is a transition from one state to another which occurs at infinitely varying rates and scales. That is why the L. Rendall's example with the writing table represents the continuation of confirming our concept regarding the transition from one state to another, in extremely unique matter and form-in other words, a writing table will gradually become soft and then turn into dust. The same happens with the animal world. But due to the endlessly varying speeds the human scope is not sufficient for every transition of flora and fauna-related transitions.

Based on the famous Einstein's formula of “mass-energy equivalence” $\mathrm{E}=\mathrm{mc}^{2}$ indicates the process of mutual mass and energy conversion. "The matter represents the parameters of a certain substance, entity, subject or object existence phenomenon". "Space is the phenomenon of a place, location or field of a certain matter existence". "Time is the phenomenon of modifying quantitative-and-qualitative states of space phenomenon existence-the matter". "Mass represents certain energy "slowed down" in its motion at the speed equivalent to the square of light speed in the vacuum". "In other words, if a certain energy phenomenon (process, event, action) is slowed down or stopped at the speed of 90,000,000,000 km/s then this energy will transform into absolute mass" [5]. It is important to understand what is primary. I believe that time is primary. It determines the process of qualitative-and-quantitative energy transformations followed by the formation of particular contents established by time, including particular specific characteristics and represented in its normal mode, i.e. every cell of a living organism is provided with a program of cellicolous, intercellular, interorgan, intersystem and integral organism living which is unique for each cell. All the processes are performed in constant endless motion made up of three "unique indivisible components: time, matter and form with permanent force of their interaction".

This is the Principle of Universal Eternity comprising an endless number of unique triangles which make up the "Energy" in its infinite motion and constant transition from one state to another" (Figure 1).

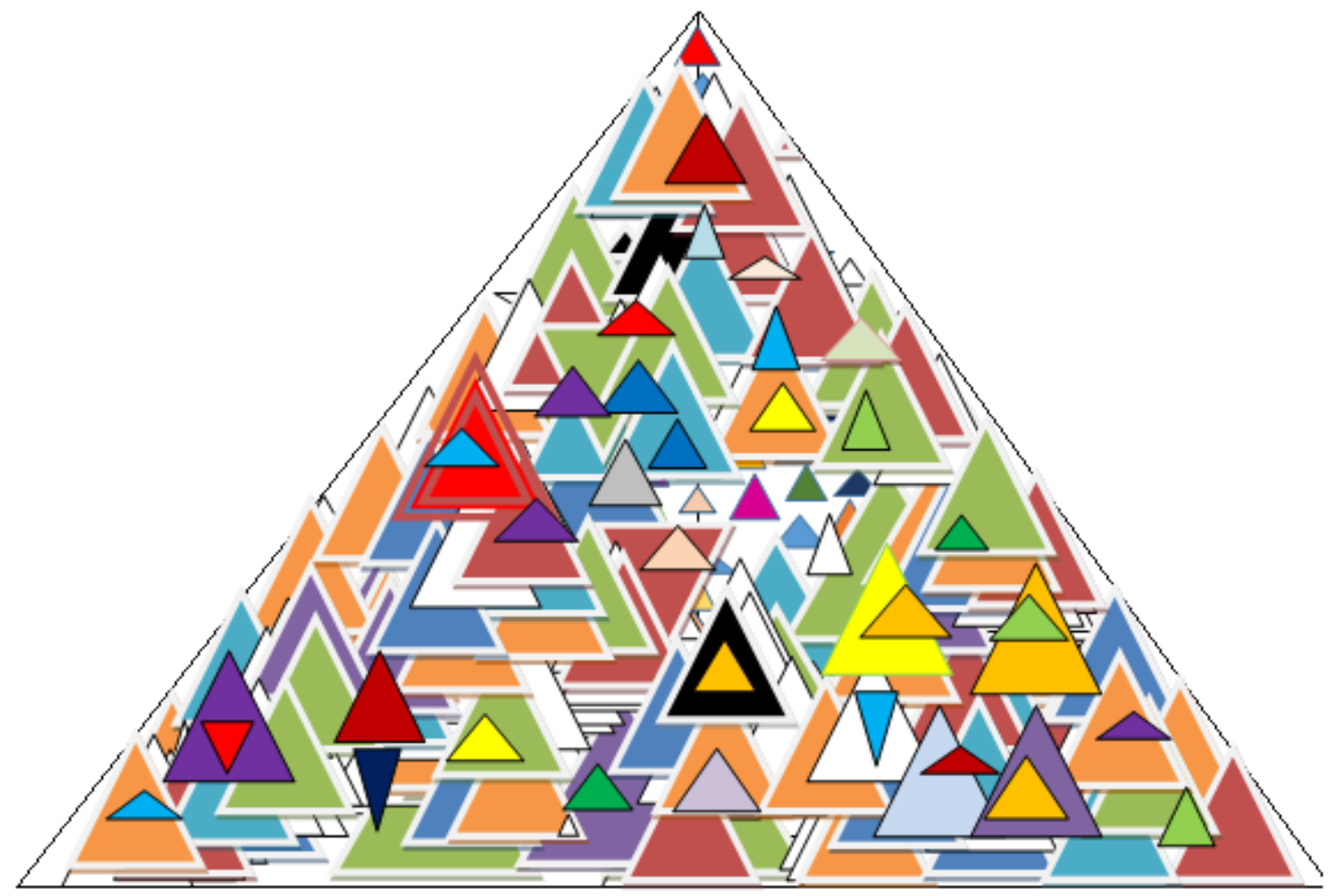

Figure 1. The principle of eternal infinity. 
Having said so, it is important to note that the scientific triplet of "time, matter and form" is unique at every moment. In the process of integral organism living at cellular and subcellular level "time, matter and form" are modified according to the law of "freedom, submission and meekness" interaction. When this principle of "interaction" is violated a disease of an organism takes place. In this regard the clinical physiotherapy task is to bring the "interaction" law into the state of harmony, i.e. "time, matter and form" are respectively identical to "freedom, submission and meekness". This means that all biological processes of an organism at cellular and subcellular levels run "freely, submissively and meekly".

Clinical physiotherapy is closely related with other fields of medicine, physics and biology.

For example, the revealing of aetiology and pathogenesis of many diseases enables not only to apply the required physical factor but also to develop a rational method as well as to use and combine it with other physical factors and pharmacological agents [1] [6]-[8].

Based on the success achieved in physics and the development of highly sensitive apparatus it became possible to determine the mechanism of low-energy physical factor effect at cellular and subcellular levels of organism [2] [9].

In this regard an experimental research on the effect of polarized polychromatic visible and infrared light emitted by medical "Bioptron" 480 - 3400 nm instrument (ENISO 9001/12.2000 certificate of conformance) was carried out.

The light passing through optical fiber cable affected main water, blood plasma and an integral organism. Infra-red spectra of irradiated samples were taken in the $4000-400 \mathrm{~cm}^{-1}$ range by using Perkin-Elmer 2000 Fourier spectroscope provided by the physical chemistry and electrochemistry institute n.a. A.A. Frumkin of the Russian Academy of Sciences (PCEI RAS, Moscow); spectra of Raman scattering for different water samples were measured with automated optical fiber spectrometers at the spectroscopy institute of the Russian Academy of Sciences (SIAS, Troitsk) and at the Scientific fiber optics centre of the Russian Academy of Sciences (Moscow), evanescent infrared skin spectroscopy was performed using a fiber-optic sensor at the Scientific fiber optics centre of the Russian Academy of Sciences (Moscow).

It is determined that the maximum effect of change in water absorption spectra is observed in the range of hydrogen bond fluctuations $\left(\sim 120 \mathrm{~cm}^{-1}\right)$. This fact may be interpreted as an increase in the size of water molecule nanoclusters connected by hydrogen bonds. Changes in spectra of valence vibrations $\left(\sim 3100 \mathrm{~cm}^{-1}\right)$ are also present on the high-frequency end $\left(\sim 3300 \mathrm{~cm}^{-1}\right)$ (Figure 2).

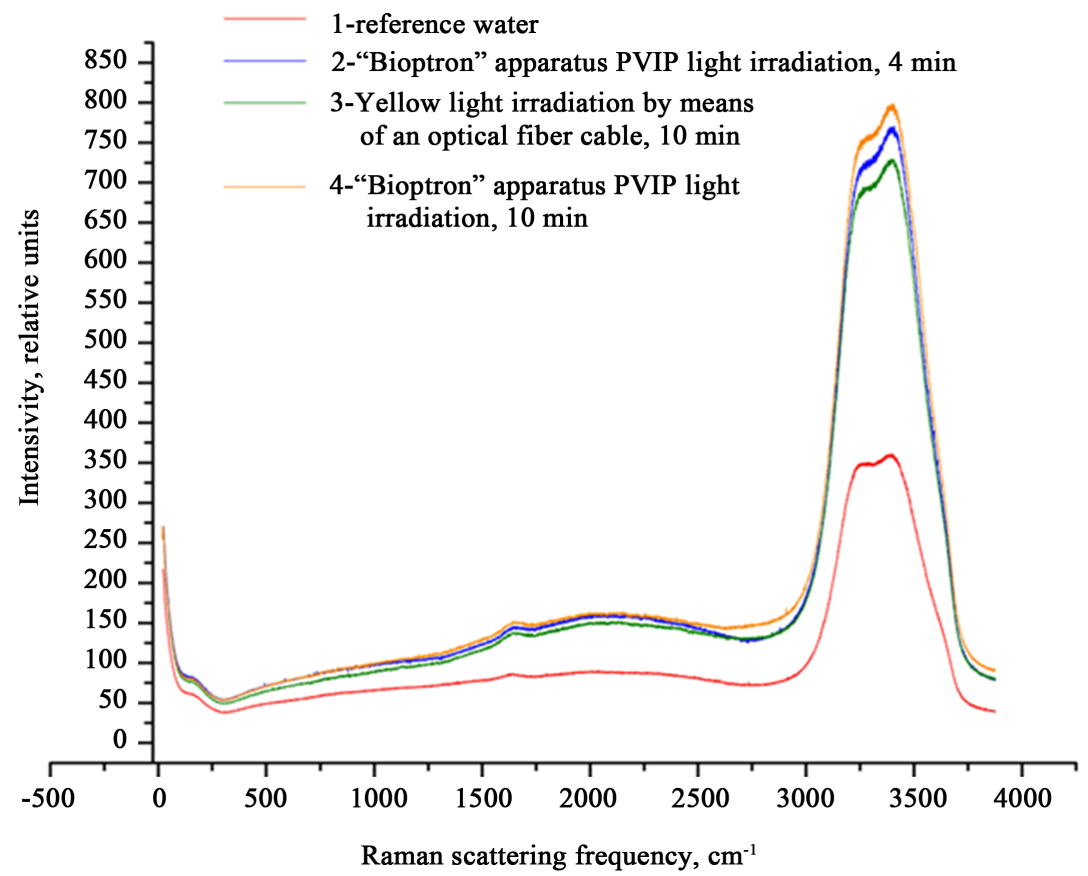

Figure 2. Area of Raman scattering (RS) spectrum of main water affected by polarized light emitted by Bioptron apparatus and by means of a fiber cable during 4 and 10 minutes. 
Radiation aftereffects after 26 and 15 minutes as well as the increase in light absorption by blood plasma 1 hour after taking a bath by the patient using preliminary irradiated water by means of polarized light were determined.

A similar effect was also displayed during the study of a volunteer's blood plasma, when one hour after taking a fifteen minute bath of water which was preliminarily irradiated for fifteen minutes it was discovered that the taken blood plasma of the volunteer absorbs light much greater than the reference plasma.

Therefore, the effect of polarized Bioptron light modifies the structures of water and blood plasma which are stable for at least one hour. It is determined that when any electromagnetic wave passes through a light guide evanescent waves are always formed around the latter. These waves are perpendicularly directed towards the light guide's outer surface and as they are moved away from it the waves damp. These previously unknown light properties were recently discovered by scientists from Japan, USA, Ukraine and Korea [10]. In this regard we conducted a range of experimental investigations (registering photons of a standing evanescent wave on skin surface after being affected with 480 - 3400 nm PVIP light).

It is established that the evanescent infrared skin spectra affected with water which is activated by light through an optical fiber cable connected from a "Bioptron" apparatus demonstrate an increase of absorption area in the spectrum range of $3200-3500 \mathrm{~cm}^{-1}$ and in the range of up to $800 \mathrm{~cm}^{-1}$. In other words there is an increase of hydration (Figures 3-6).

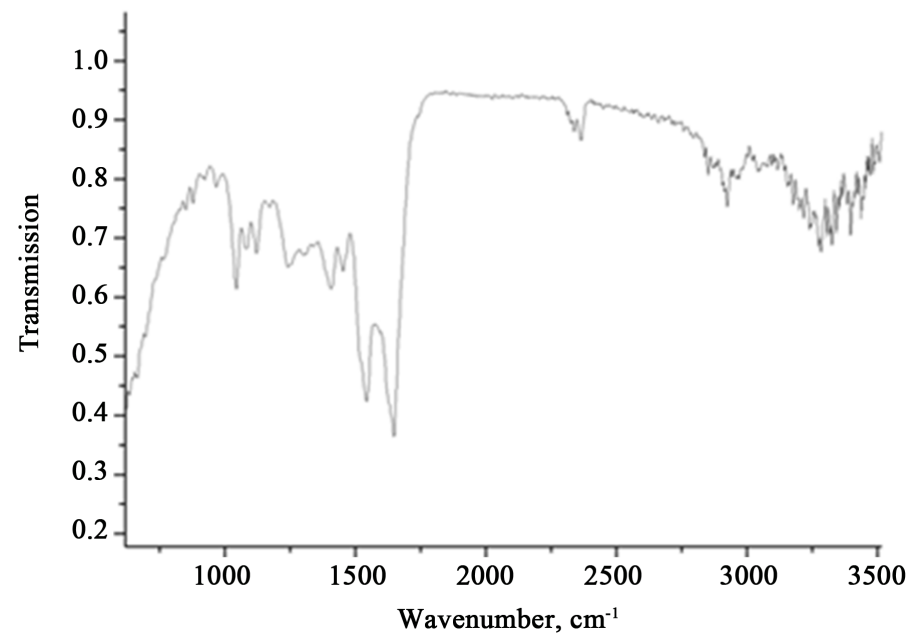

Figure 3. Infrared skin spectrum after affecting the hand with yellow light by means of an optical fiber cable during ten minutes.

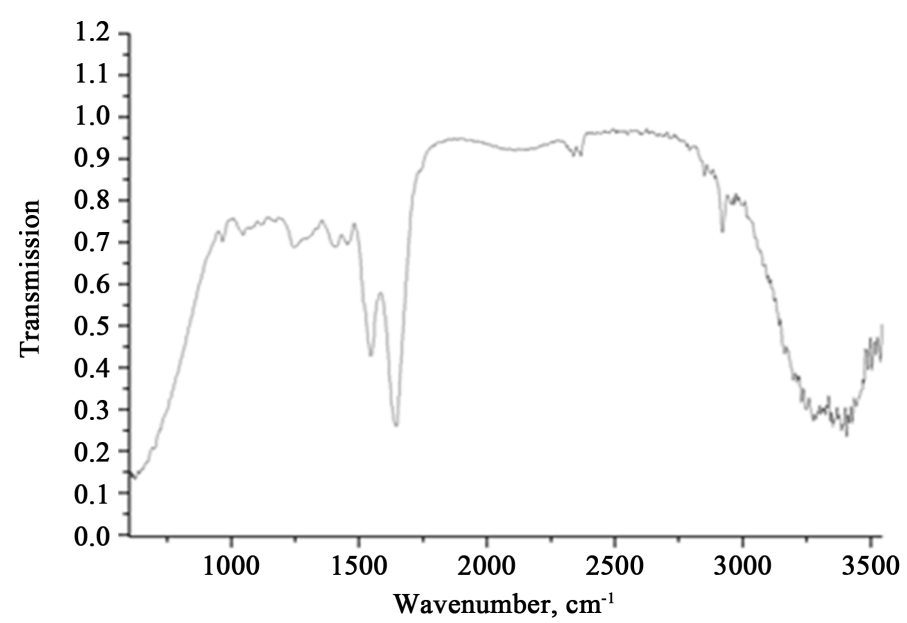

Figure 4. Infrared skin spectrum after dipping the hand into water irradiated with yellow light by means of an optical fiber cable during ten minutes. 


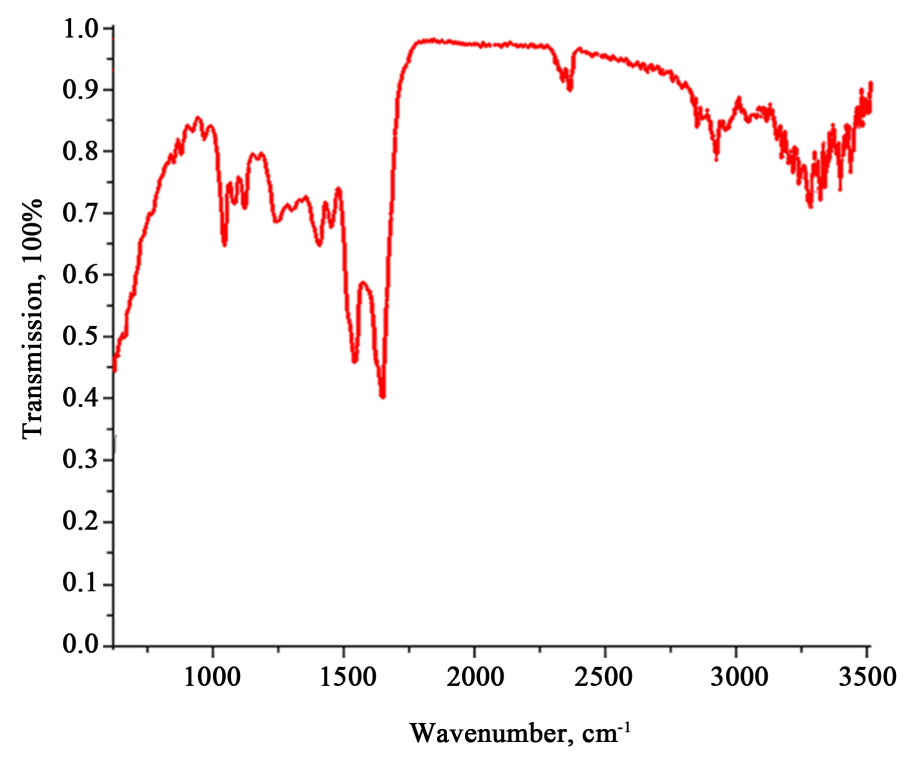

Figure 5. Infrared skin spectrum after affecting the hand with polarized light emitted by "Bioptron” apparatus during 10 minutes.

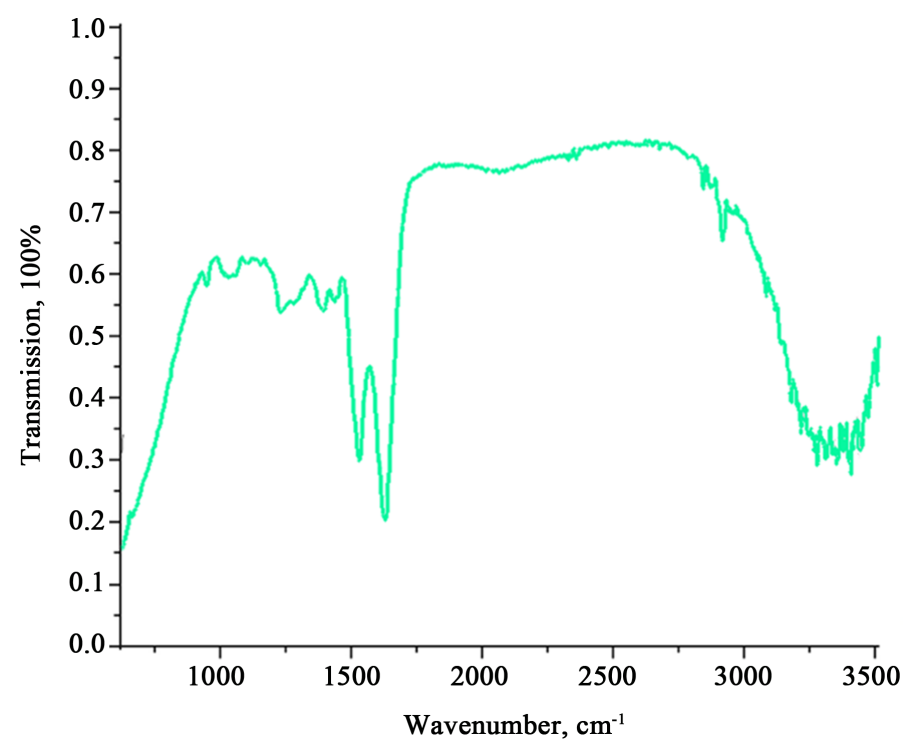

Figure 6. Infrared skin spectrum after dipping the hand into water irradiated with "Bioptron" light during 10 minutes.

As a result there is a reason to apply phototherapy using "Bioptron" apparatus-through an optical fiber cable both under direct influence and indirectly through water- to various locomotion system and skin-related diseases.

Based on the described experiments it is possible to make a conclusion that photoexcitation of water using the "Bioptron" source really results in a change of water structure. This became possible due to the absorption of source-provided irradiation inside water which stimulates the growth of the size of water nanoclusters connected together by means of a hydrogen bond which became possible as a result of revealing uncommon light properties by the above mentioned scientists (the emergence of evanescent waves) and the creation of "scanning tunneling microscope" (Stephen Mendak and his Germany-based colleagues created a working tool which is capable to reconstruct two-dimensional images of three-dimensional nanoscale objects using the light of visible frequency and the nearest infrared spectrum. The device is based on "superlenses" made of thin flat silver bars in the form of a tube with a central opening of about 2 microns. Evanescent waves pass outwards through the 
tube's layers from its inner surface perpendicularly to the circumference and during such movement there is a primary image magnification, i.e. the "scanning tunneling microscope") [10].

It is possible to state that "in relation to molecules the supermolecules represent the same thing that the molecules represent in relation to atoms provided that the role of covalent bonds in supermolecules is played by intermolecular interactions" [3].

Therefore, supermolecules represent the next level of matter organization's complexity following elementary particles, nuclei, atoms and molecules [3].

In 1982 a Russian scientist and a laureate of the Lenin Prize in physics Gurgen Ashotovich Askaryan experimentally proved "an increase in the transmission of laser and other types of irradiation through soft turbid physical and biological media”.

It is well-known that one of the interesting applications is based on its stimulating effect on regenerative and immune processes within cells and tissues of an organism, particularly in view of the resonant nature of absorptions and stimulations (one of the absorption fields is close to the He-Ne-laser generation line with a 0.63 micron wavelength). It is precisely these processes that link the use of such lasers with the treatment of trophic ulcers, nonhealing wounds, stomatitis, polyneuritis and other pathological processes occurring within the outside layers [11].

In his first experiment the author used a layer of foam-rubber modeling a highly scattering medium. He used a continuous action 15 - 20 mW LG-75 He-Ne-laser as an experiment. Its ray was incident on the examined layer pressed in by a cylinder or a tube. The layer was located on top of a fixed glass plate and squeezed between the plate and the cylinder. The thickness of locally compressed layer-distance between the introduced side of the cylinder and the glass plate-was measured. Diffused light spot at the scattering light exit was registered with a photo camera. Diffused light spot at the exit depends on the thickness of compressed layer. The smaller the thickness of compressed layer the larger the diffused light spot at the exit [11].

In his second experiment G.A. Askaryan studied the change of light penetration through the tissues of human body. A palm was used as a scattering layer. The He-Ne-laser ray was incident on the centre of the palm. The palm thickness at its central part was $2.7 \mathrm{~cm}$, when applied slight algesic pressure the thickness was $2 \mathrm{~cm}$.

When the light was incident on the back side of right hand and the palm was pressed with a glass stick a sudden intensification of light transmission was observed from the opposite side. When the palm of left hand was pressed on the plexiglass cylinder across which the light was passed through an intensified light transmission was observed from the opposite side. The same intensification was already observed with 2.7 - $2 \mathrm{~cm}$ pressing, i.e. the blooming effect when pressing with palm was much greater compared to using foam-rubber [11].

The author links this with sideward extrusion of blood and tissues. However it is worth noting during his experiment that even the thicker parts of the palm—flesh with blood and the area near the finger bones — are more permeable by light and more subject to blooming by means of pressing as compared to its central part. It is interesting to note the relaxation of blooming: when pressing is gone the blooming does not disappear immediately - the pressing mark lets the light through for another $1-3$ seconds. This is confirmed by our abovementioned experiments—-the aftereffects of irradiation (see Figure 7 and Figure 8).

Without general theory it is extremely hard to describe a course in physiotherapy harmoniously and systematically. This cannot be neglected at the time of scientific and technical advance along with health service reformation requiring the training of specialists with high expertise and sufficient professionalism" [8].

In his unique "Outlines of general therapy" book (1994) V.S. Ulaschik named the main reasons for inhibiting the elaboration of general physiotherapy theory:

- insufficiently developed ideas about the mechanism of medical physical factor influence on the organism due to a missing outstanding creative personality, a generator of ideas, a leader since the following outstanding representatives of Russian physiotherapy have passed away: A.N. Obrosov, A. P. Speranskiy, A.R. Kirichenskiy, A.P. Parfionov, I.A. Piontkovskiy and D.A. Markov. They have done a lot for the development of general theory but failed to complete it;

- low prestigiousness of theoretical developments when compared to experimental and clinical research of particular physical factors;

- inadequate link between not only the proposed hypotheses and theories but also the research on balneology and physiotherapy in general and the achievements of theoretical medicine-first of all, physiology, pathology and biophysics; 


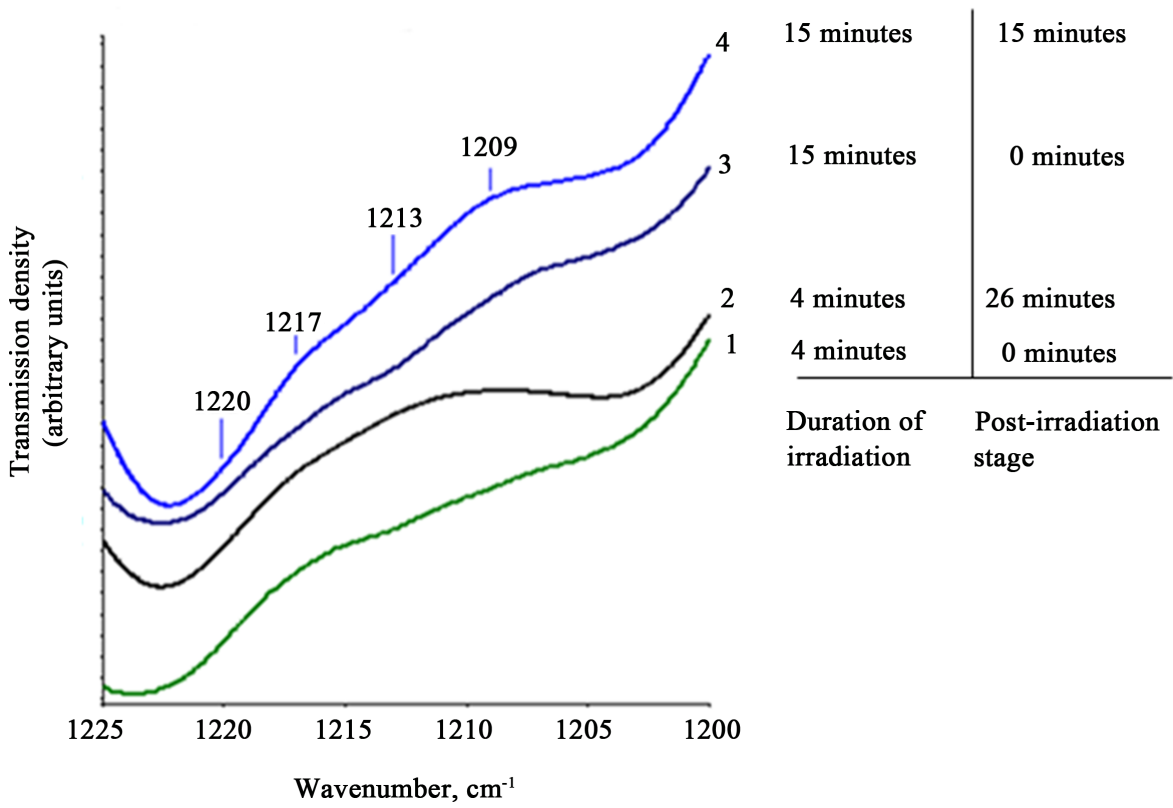

Figure 7. The area of infrared spectrum of main water affected by polarized light emitted by "Bioptron": 1: after four-minute water irradiation; 2: 26 minutes after the four-minute water irradiation; 3: after fifteen-minute water irradiation; 4: 15 minutes after the fifteen-minute water irradiation.

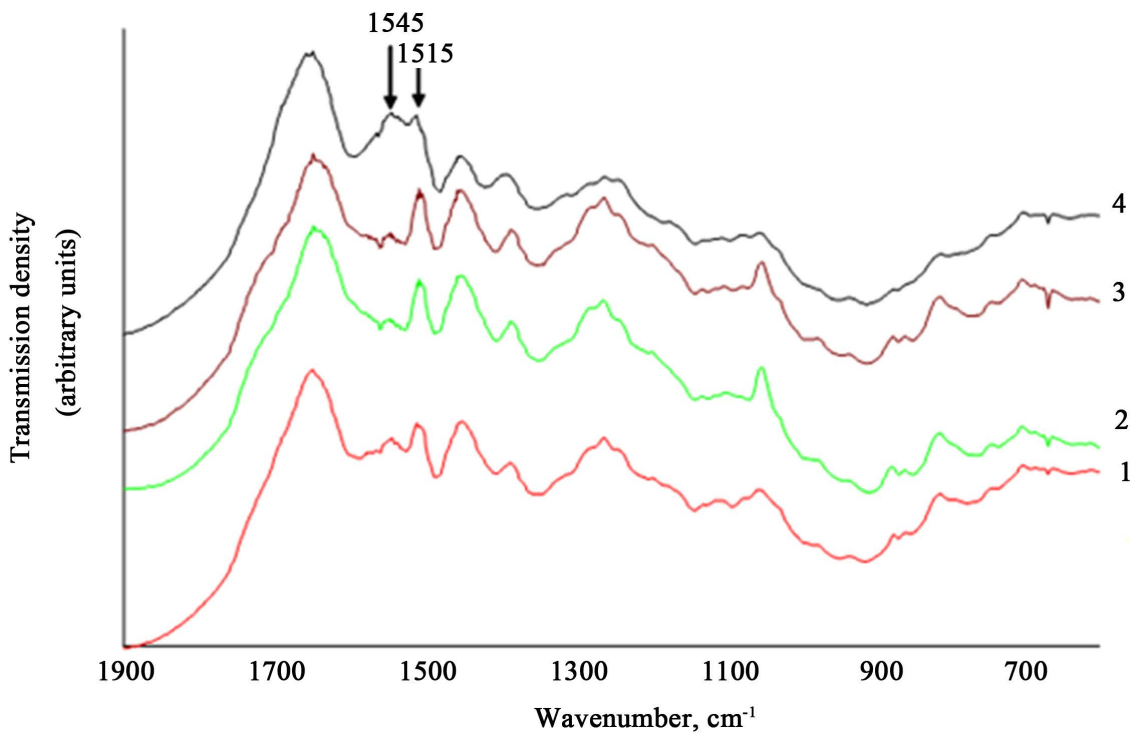

Figure 8. Infrared spectra of blood plasma after being affected with polarized "Bioptron" light: 1. blood plasma spectrum before irradiation (reference); 2. blood plasma spectrum after Bioptron PVIP light irradiation in a Petri dish during 4 minutes; 3. plasma spectrum after Bioptron PVIP light irradiation in a Petri dish during 15 minutes; 4 . plasma spectrum 60 minutes after a fifteen-minute bath taken by the patient (the water was preliminarily irradiated with PVIP light during fifteen minutes).

- insufficient theoretical generalizations caused by the lack of experimental research in both physiotherapy and balneology. In this regard V.S. Ulaschik quotes P.L. Kapitsa: "High quality of an experiment is a requirement for sound science development".

"To construct general theory of physiotherapy it is necessary to study doze dependencies, specificity and selectivity of the effect, threshold organism sensitivity to physiotherapeutic influences, etc. The issue of studying informational mechanism of medical physical factor influence is extremely pointedly posed” [8]. 


\section{Research Results and Their Discussion}

The analysis of experimental research results made it possible to confirm that light passing through a fiber- optic cable activates tap water, blood plasma and the entire organism. The exposure consequences effect after 26 and 15 minutes has been observed as well as the increase in light absorption through blood plasma 1 hour after the patient had a bath with water exposed to polarized light.

Evanescent infrared skin spectra after being exposed to water activated with yellow light through a fiber-optic cable show an increase in absorption areas within spectral ranges from 3200 to $3500 \mathrm{~cm}^{-1}$ and in the range up to $3000 \mathrm{~cm}^{-1}$, i.e. it leads to a change in water structure: the growth of dimensions of water nanoclusters linked with hydrogen bond, which became possible due to the identification of the unusual properties of light (the appearance of evanescent waves) and due to the creation of a "scanning tunneling microscope" by German scientists.

It should be noted that results of experiments by G.A. Askaryan to the effect that with pressure removed, manifestation relaxation intensity does not disappear at once-pressure trace permits light through itself for another 1 to $3 \mathrm{sec}$. This is a confirmation of your experiments—an aftereffect (Figure 7 and Figure 8). Experiments by G.A. Askaryan of "increase of the laser and other radiation passage through turbid physical and biological media confirm our concept that the entire organism consists of a set of "biological scanning tunneling microscopes", that is the passage of light through the entire depth of a palm is not associated with the displacement of tissue blood sideways as stated by the author of the experiment, but evanescent waves are formed layerwise in the palm depth, followed by their leaving the palm from its opposite side.

One more fact in favor of our concept is the experiment author's conclusion that the effect of translucence is much stronger with a palm pressed than with the use of foam plastic, and even thicker parts of the palm, flesh with blood and phalanges areas are more exposed to translucence than the palm central part.

Radiation aftereffects after 26 and 15 minutes as well as the increase in light absorption by blood plasma 1 hour after taking a bath by the patient using preliminary irradiated water by means of polarized light were determined.

I. Efficiency of using physical factors in complex patient therapy depends on execution of the following tasks of clinical physiotherapy:

1. Clinical tests of new physiotherapeutic methods and procedures.

2. Clinical research and revaluation of old physiotherapeutic methods and procedures.

3. Developing an effective and safe physiotherapeutic method.

4. Organizing information services and consultative aid provided to different specialists.

5. Training students and doctors.

II. In this regard I suggest creation of a new seven-principles-classification of using physical factors in complex patient therapy.

Principle one:

According to the source energy

1. Natural:

- Solar energy;

- Cosmic energy;

- Temperature (air space, water: rivers, lakes, seas, mineral waters, therapeutic muds);

- Human endobioenergy:

- Energy system's communication apparatus (energy centers);

- Superstable acousto-electromagnetic waves (solitons) contained in human DNA;

- Flora energy;

- Exobioenergy (leeches, bees, etc.).

2. Artificially produced energy:

Electrical; Magnetic; Light; Thermal; Water power; Mechanical; Radiowave; Energies produced using air pressure and gases; Fermi-Pasta-Ulam generator (for generating solitonic electroacoustic fields).

Principle two

According to the most important areas of using medical physical factors

1. With medical purposes: Natural factors; Preformed factors.

2. Rehabilitation area: Natural factors; Preformed factors. 
3. Physioprophylaxis: Primary prophylaxis-mainly by using natural factors; Secondary prophylaxis-mainly by using preformed factors.

4. Physiodiagnostics: Electrodiagnostics; Electropunctural diagnostics; Resonant laser spectroscopy of Radiowave radiator of objects including chromosomes, living cells and tissues; Biomolecular-opto-radio-electronic computer systems - analogues of a chromosomal biocomputer of an organism; infrared spectroscopy in the range of $4000-400 \mathrm{~cm}^{-1}$ using Perkin-Elmer 2000 Fourier spectroscope; Raman spectroscopy; Evanescent spectroscopy.

Principle three

According to physicochemical basis of physical factor influence:

1) Thermogenesis; 2) Ionic shifts; 3) Formation of free physical agents; 4) Generation of free radicals; 5)

Conformational changes.

Principle four

According to energy type and action mode

1. Constant electrical currents: Continuous; Pulse.

2. Variable electrical currents: Low frequency; Medium frequency.

3. Electrical field: Constant; Pulse; High and ultrahigh frequency.

4. Magnetic field: Constant; Pulse; Low frequency; High frequency.

5. Electromagnetic radiation of radio-frequency range: Ultrahigh frequency (S-band and L-band); Extremely high frequency (EHF).

6. Electromagnetic radiation of optical range: Infrared; Visible; Ultraviolet (DUV, SUV, KUV); Monochromatic, coherent.

7. Mechanical energy:

- Mechanical stresses (therapeutic massage, manual therapy, acupuncture);

- Mechanical oscillations: Vibration; Ultrasound.

- Aerial space factors:

a) Atmospheric pressure: Alternate (cycling); Lower; Higher; Partial (low $\mathrm{PO}_{2}$ and high $\mathrm{PO}_{2}$ ); High $\mathrm{PO}_{2}$ and $\mathrm{PHe}$ (oxygenoheliotherapy); High $\mathrm{PCO}_{2}$ (carbogenotherapy).

b) Aerons;

c) Aerosols.

Principle five

According to the mechanism of physical factor influence

Principle six

According to the ability to interact with bioenergy of cells and tissues

Principle seven

According to the method of injecting pharmacological agents into the organism

\section{Conclusions}

It can therefore be concluded that new, previously unknown mechanisms of weak electromagnetic wave impact on the integral organism and methods of their registration were discovered. This means that the integral organism is made up of multiple "scanning tunneling biomicroscopes of the near field" since it conforms to the description provided by Stephen Mendak's apparatus from Germany. We believe that when electromagnetic waves affect the integral organism evanescent waves are created at the opposite side of all capillaries, vessels, nerve trunks and rootlets. These waves are the multiple "scanning tunneling biomicroscopes" whereas the registration of PVIP light's effect on the organism is performed by means of a "scanning tunneling photonic microscope", see Figure 9 (the Fermi-Pasta-Ulam phenomenon of "reverse energy return").

In this regard it is reasonable to use the phototherapy with "Bioptron" in musculoskeletal system disorders and dermal diseases through an optical fiber cable both at direct application and at indirect application through the water.

It must be supposed that every cell of a living organism is provided with a unique program of everlasting motion at an individual speed applied to all the processes of their activities in particular and altogether (i.e. a transition from one state to the consecutive one...).

The uniqueness and the indivisibility of "time, matter and form" along with the forces of their interaction 


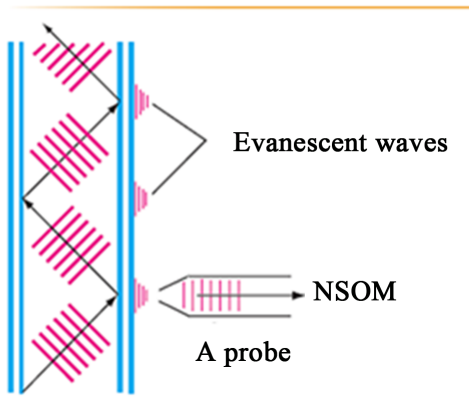

A light guide with a wave advancing along it

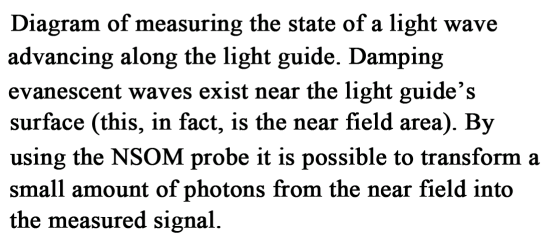

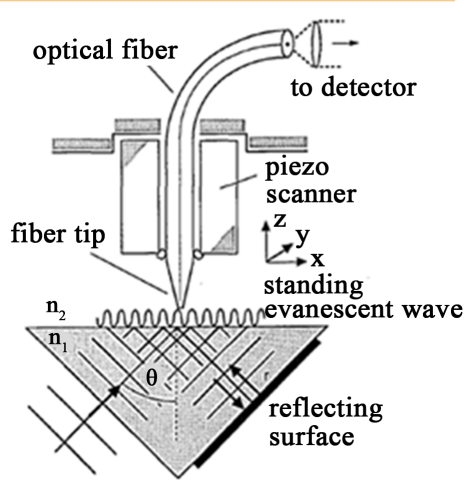

Diagram of registering photons of a standing evanescent wave bonded with top prism surface using a scanning tunneling photon microscope.

\section{Figure 9. Evanescent waves.}

during constant everlasting motion represent the Law of Universal Eternity denoted as a triangle filled with small multicolored triangle shapes of different sizes.

The results of our researches enable us to define the clinical physiotherapy:

"The science that studies power-and bio-informational systems of organism, possibilities of their correction at subcellular level with the use of physical factors in order to stabilize disordered biological processes of organs and systems is called clinical physiotherapy".

\section{Recommendations}

Practically the clinical physiotherapy must solve the following issues:

- choosing the physiotherapeutic method for medical treatment of a particular patient at the moment of his clinical condition judgment;

- defining the most suitable physical factor parameters, their application mode and combinations with other physical factors;

- choosing the ways of physical factor influence;

- observing the effect of physiotherapeutic factors;

- preventing and eliminating by-reactions.

Prior to treatment prescription a physical therapist should answer the following questions.

1. What particular changes in the patient's condition does he want to obtain?

2. Which physical factors may provide the desired effect?

3. Which physiotherapeutic procedure is mostly suitable to this patient?

4. In which combination and order should the selected physiotherapeutic method be applied along with other physical factors?

The effectiveness and the safety of physiotherapy eventually depend on the doctor's ability to evaluate all these factors correctly. It is necessary to be based on the deep knowledge of both clinical medicine and experimental physiotherapy.

Make good use of underwater phototherapy: yellow light through the optical fiber cable as high efficiency method of physiotherapy.

Actively participate in discussion of the new supposed classification of physical factors in complex patient therapy.

\section{References}

[1] Bogolyubov, V.M. and Ponomarenko, G.N. (1997) General Physiotherapy. 2nd Edition, Revised. M., SLP, St. Peters- 
burg, $480 \mathrm{p}$.

[2] Betskiy, O.V., Golant, M.B. and Deviatkov, N.M. (1988) Millimeter Waves in Biology. M.,.

[3] Lehn, J.-M. (1998) Supramolecular Chemistry. "Nauka”, Novosibirsk.

[4] Rendall, L. (2014) Knocking on Heaven's Door: A Scientific Look at the Arrangement of the Universe. Translation from English, M.: Alpina non-fiction, 518 p.

[5] Einstein, A. (1967) Tractate Collection IV. Articles, Reviews, Letters. Evolution of Physics. "Nauka” Publishers, Moscow.

[6] Clinical Physiotherapy/Under the Editorship of V.V. Orzheshkovskiy, K. Zdorovya, 1985, 446 p.

[7] Klyachkin, L.M. and Vinogradova, M.N. (1982) Physiotherapy. M.:Meditsina, 272 p.

[8] Ulaschik, V.S. (1994) Outlines of General Physiotherapy. Minsk. Nauka I tekhnika, 198 p.

[9] Volkov, E.S. and Vlyalko, V.I. (1989) Electricity Serves Health. K.: Zdorovya, 88 p.

[10] Andryushin, E.A. (2007) The Power of Nanotechnologies: Science \& Business. In: Andryushin, E.A., Ed., B.M.: Success of Physics, 159 p: il; Bibliogr.: 155-157.

[11] Batanov, G.M., Bolotovskiy, B.M., Grigoryan, S.S., Kossiy, I.A. and Sokolov, I.V. (1998) In Memory of G.A. Askaryan.M.: Znak, 376 p. 